\title{
Spatial disparities and associated factors of Under- five diarrhea disease in llubabor Zone, Oromia Regional State, Ethiopia
}

Ebsa Gelan ( $\sim$ ebsagelan@gmail.com )

Mettu university

Mulata Worku

Mettu university

Azmeraw Misganaw

Mettu university

Dabala Jabessa

Mettu university

Research Article

Keywords: Under-five diarrhea, Spatial Autocorrelation, Bayesian Geo-additive Regression Model

Posted Date: January 7th, 2022

DOI: https://doi.org/10.21203/rs.3.rs-1169274/v1

License: (c) (i) This work is licensed under a Creative Commons Attribution 4.0 International License.

Read Full License 
Spatial disparities and associated factors of Under-five diarrhea disease in Ilubabor Zone, Oromia Regional State, Ethiopia.

Ebsa Gelan', Mulata Worku², Azmeraw Misganaw ${ }^{3}$ and Dabala Jabessa 4

${ }^{1}$ Departement of statistics, College of Natural science, Mettu university, Mettu,Ethiopia

Email:ebsagelan@gmail.com

Phone number: $+\mathbf{2 5 1 9 6 4 3 1 5 3 8 6}$

${ }^{2}$ Departement of statistics, College of Natural science, Mettu university, Mettu,Ethiopia

Email:mulataworku@gmail.com

Phone number: +251928651325

${ }^{3}$ Departement of statistics, College of Natural science, Mettu university, Mettu,Ethiopia

Email: azmerawmisganaw@yahoo.com

Phone number: +251906397753

${ }^{4}$ Departement of statistics, College of Natural science, Mettu university, Mettu,Ethiopia

Email: debelagabessa123@gmail.com

Phone number: +251921837085

\section{Abstract}

Diarrhea is commonly a sign of an infection in the intestinal tract that is caused by different bacteria, virus and parasitic entities. It is one of the leading causes of child mortality worldwide, especially in sub-Saharan Africa countries including Ethiopia. The main objective of this study was to identify spatial disparities and associated factors of under- five diarrhea disease in Ilubabor zone, Oromia regional state, Ethiopia. The study has been conducted in Ilu Aba Bor zone of entire districts and the data is basically both primary and secondary which were obtained from each woreda health office of Ilu Aba Bor zone and corresponding mother or care givers of sampled child. Spatial disparities of under-five diarrhea were identified using global and local measures of spatial autocorrelation. Geo-additive regression model was used to identify the spatial disparities and associated factors of under-five diarrheal disease. The value of global and local measures of spatial autocorrelation shows that under-five diarrheal disease varies according to geographical location and shows significant positive spatial autocorrelation. The results of Geo-additive regression model showed that statistically significant relationship between underfive diarrhea disease and independent variables. There is evidence of significant under-five diarrheal disease clustering in Ilu Aba Bor zone, southwest Ethiopia. Model based data analysis showed that there is significant relationship between Under-five diarrhea and covariates 
(mother's age, mother's education, source of drinking water, quality of toilet facility, DPT 3 vaccination, Polio 3 vaccination and household wealth index.).

Keywords: Under-five diarrhea, Spatial Autocorrelation, Bayesian Geo-additive Regression Model

\section{Background of the study}

\section{INTRODUCTION}

The world health organization defines diarrhea as the passage of three or more loose or liquid stools per day in a period not exceeding 14 days. Diarrhea is one of the leading causes of child mortality, mostly in children less than 5 years of age living in low and middle-income countries [16]. Globally 530,000 children under five years old are dying in a year due to diarrheal disease and diarrhea is the second leading cause of mortality in under- five children next pneumonia which is responsible for one in nine children mortality [17]. In Africa, especially in sub-Saharan Africa countries diarrheal disease accounts for over $88 \%$ of deaths in children below five years old in 2015[17].In Ethiopia, childhood morbidity and mortality remain high due to the burden associated with highly prevalent diseases such as diarrhea, fever, cough, malaria and HIV-AIDS. For instance, diarrhea contributes to more than one in every ten (13\%) child deaths in Ethiopia [19].Diarrhea is commonly a sign of an infection in the intestinal tract that is caused by different bacteria, virus and parasitic entities [18] and characterized by abnormally loose or watery stools. Diarrhea remains the leading cause of morbidity and mortality in children under 5 years old worldwide. Particularly diarrhea disease causes $8 \%$ of deaths among children under age five globally [20].The burden is disproportionately high among children in low and middle income countries. Young children are especially vulnerable to diarrheal disease and a high proportion of the deaths occur in the first 2 years of life. Worldwide, the majority of deaths related to diarrhea take place in Africa and South Asia. Nearly half of deaths from diarrhea among young children occur in Africa where diarrhea is the largest cause of death among children under 5 years old and a major cause of childhood illness [5]. According to the UNICEF report in 2016, approximately 5.6 million children under age of five die every year, which is decreased from over 12 million in 1990. $80 \%$ of deaths are from sub-Saharan African like Ethiopia. Regardless of the noticeable improvement in the reduction of under-five death in Ethiopia, childhood diarrhea is still the leading cause of death. In Ethiopia few studies are conducted on diarrhea to find risk factors associated with under- five diarrhea disease $[13,15]$. However they didn't include spatial 
dependence in their work, which gives incorrect result on the estimation of parameter, hence most of health event data are spatially correlated. However finding a spatial disparity is very important to design and monitor effective intervention programs to reduce the prevalence of diarrhea. The basic research questions are: Does the spatial distribution of diarrhea spatially random or clustered in districts of Illubabor zone, which district is/are under cold spot and which district is under hot spot and among study variables which predictor variables significantly affect under five diarrhea diseases in this study area. The general objective of this study was to identify spatial disparities and associated factors of under- five diarrhea disease in Illubabor zone, Oromia regional state, Ethiopia.

\section{The significance of the study}

The organizations as well as individuals who work in this area get a clue on the spatial disparities

of diarrhea in the districts of Illubabor zone. The other basic significance of this study was to further assist other researchers interested in explanatory spatial data analysis and they may use it as a benchmark for their future works in identifying spatial distribution of disease, therefore, researchers would benefit by getting familiar with this method and may further help in advertising explanatory spatial data analysis, Therefore, the result of this study has the following importance:

$>$ It enables government and other concerned organizations for strategic planning to reduce the prevalence of under -five diarrhea disease.

$>$ Provide information to researchers for further studies on explanatory spatial data analysis and use different statistical model.

Help to identify factors that affect diarrhea prevalence in this study area.

Help to identify woredas under hot spot and cold spot.

\section{Methodology}

Source of the data: For this study both secondary and primary source of data were used. Secondary data (under-five diarrhea cases counts for each woreda) was taken from each woredas of Ilubabor zone for the purpose of spatial pattern analysis and community based data(primary source data) was taken from corresponding mother or care givers of sampled child for the purpose of associated factors of under-five diarrhea analysis. For the data collection the researchers used both questionnaire and face to face interview as data collection tools. The 
questionnaire was distributed for the corresponding mother or care givers of sampled child by translating it to local language (Afaan oromoo) in order to get correct information. Variable of the study: The variables included in this study were listed as follows:

Dependent variable: The primary outcome variable was the reported occurrence of diarrhea. i.e. If the child had diarrhea in the last two weeks including the last 24 hours prior to data collection time. Therefore, the response variable was dichotomous in nature i.e. children diarrhea status $(0=$ no, $1=$ yes (child had diarrhea))

Independent Variables: mother's age, mother's education, mother's work status, source of drinking water, mother's place of residence, quality of toilet facility, DPT 3 vaccination, Polio 3 vaccination, age of the child and household wealth index.

\section{Method of Data Analysis}

\section{Spatial data Analysis}

Spatial data analysis is defined as an analysis that involves the accurate description of data relating to a process operating in space, the exploration of patterns and relationship in such data, and the search for explanation of such patterns and relationships [3].

Therefore in this study exploratory spatial data analysis like Moran's I and Geary's C was used to detect the presence of spatial dependency (association) in our data and Bayesian Geo-additive regression model is used to identify the determinants of prevalence of diarrhea with spatial disparities. Spatial dependence indicates that near places are more likely to be related than distant ones and usually most geographical patterns of interest involve groupings of similar values in clusters.

\section{Moran's I}

Moran's I Global measures summarize spatial association with respect to the whole region. The Moran I values ranged from -1 to +1 . A positive spatial autocorrelation $(+1)$ referred to a map pattern where geographical features of similar value tended to cluster together, a negative spatial autocorrelation showed a map pattern in which geographical units of similar values were scattered throughout the map and a statistically insignificant spatial autocorrelation depicted a random distribution. The general formula for computing Moran's I is:

$$
I=\frac{N}{\sum_{i} \sum_{j} w_{i j}} \frac{\sum_{i} \sum_{j} w_{i j}\left(y_{i}-\bar{y}\right)\left(y_{j}-\bar{y}\right)}{\sum_{i}\left(y_{i}-\bar{y}\right)^{2}}
$$


Where $N$ is the number of spatial units indexed by $i$ and $j ; y$ is the variable of interest; $\bar{y}$ is the mean of $y$; and $w_{i j}$ is an element of a matrix of spatial weights. The observed value of $I$ can be compared to its distribution under the null hypothesis of no spatial autocorrelation or no clustering i.e. when the values of $y i$ are independent of the values $y j(i \neq j)$ at neighboring locations. This is equivalent to say that under the reference null distribution, data are randomly distributed over locations. Therefore, inference can be based on the standardized version of I, namely: $Z(I)=\frac{I-E(I)}{\sqrt{\operatorname{var}(I)}}$

The expected value of Moran's I under the null hypothesis of no spatial autocorrelation is

$$
E(I)=\frac{-1}{N-1}
$$

Its variance equals $\operatorname{var}(I)=E\left(I^{2}\right)-(E(I))^{2}=\frac{N^{2}(N-1) S_{1}-N(N-1) S_{2}-2 S_{0}^{2}}{(N+1)(N-1) S_{0}^{2}}$

Where $S_{0}=\sum_{i \neq j}^{n} w i j, S_{1}=\frac{1}{2} \sum_{i} \sum_{j}\left(w_{i j}+w_{j i}\right)^{2}, S_{2}=\sum_{i}\left(\sum_{j} w_{i j}-\sum_{j} w_{j i}\right)^{2}$

In this study, the global Moran's I test statistic is used to test the null hypothesis:

Ho: No significant clustering of diarrhea prevalence (no spatial auto correlation) in the entire study region. The mean found by Moran's I coefficient analysis is used to identify the presence of spatial autocorrelation by comparing Moran's I calculated with the tabulated value.

\section{Geary's C}

The Geary C statistic is useful in identifying local patterns of health event distribution (distribution of diarrhea prevalence in case of this study).Geary's $\mathrm{C}$ interactions are not the cross product of the deviations from the mean, but the deviations in intensities of each observation location with one another.

Geary's C can be computed as follows:

$$
C=\frac{(N-1) \sum_{i=1}^{N} \sum_{j=1}^{N} w_{i j}\left(y_{i}-\mathrm{y}_{\mathrm{j}}\right)^{2}}{2\left(\sum_{i=1}^{N} \sum_{j=1}^{N} w_{i j}\right) \sum_{i=1}^{N}\left(y_{i}-\bar{y}\right)^{2}}
$$

Usually the values of $\mathrm{C}$ range between 0 and 2.Values of $\mathrm{C}$ between 1 and 2 indicate presence of negative spatial autocorrelation while values between 0 and 1 indicate presence of positive 
spatial autocorrelation and a value of 1 showing a random pattern. Moran's I gives a more global indicator, whereas the Geary's coefficient is more sensitive to differences in small neighborhoods. Testing the significance is done by using the standardized version of $\mathrm{C}$, namely:

$$
Z(C)=\frac{C-E(C)}{\sqrt{\operatorname{var}(C)}}
$$

With $E(C)=1, \operatorname{var}(C)=\frac{\left((2 S 1+S 2)(n-1)-4 S O^{2}\right)}{2(n+1) S 0}$

\section{Bayesian Geo-additive Regression Model for Binary Spatial data}

A common way to build regression models extending the classical linear model for Gaussian responses to more general situations such as binary responses are generalized linear models, originally introduced [14]. While being flexible in terms of the supported response distributions, generalized linear models obey rather strong assumptions considering the linearity of the influence of covariates and the independence of the observations. However in most practical situation, we are facing at least one of the following problems in which generalized linear model is not appropriate to fit data:

* For the continuous covariates in the data set, the assumption of a strictly linear effect on the predictor may not be appropriate.

* Observations may be spatially correlated.

* Heterogeneity among individuals or units may not be sufficiently described by covariates because of the hierarchical structure of the data.

To overcome these difficulties a model with predictors that contain spatial effect and non-linear effect of metrical covariate called Geo-additive regression model is proposed[9].Geo-additive model is one class of structural additive regression (STAR) model which based on the framework of Bayesian generalized linear models[11, 4].

\section{Model building}

Consider regression models, where observations $\left.\left(y_{i}, x_{i}, w_{i}\right) ;\right) ; \mathrm{i}=1 \ldots \mathrm{n}$ on response $y_{i}$, a vector $x_{i=}=\left(x_{1} \ldots \ldots \ldots x_{p}\right)$ 'of metrical covariates (continuous covariate), spatial covariates (district or woreda) in case of this study and a vector $w_{i}=\left(w_{1}, \ldots \ldots w_{r}\right)^{\prime}$ Of further covariate, in which categorical variables are often given.

Where, the response $\mathrm{Yi}$ is a binary indicator of the response variable 


\section{i.e. Yi Binomial $(n i, \pi i)$}

The generalized additive modeling framework assumes that, given $x_{i}$ and $w_{i}$, the distribution of the response yi belongs to an exponential family, with mean $\mu_{i}=\left(y_{i} \mid x_{i}, w_{i}\right)$ linked to an additive semi-parametric predictor $\mu_{i}=h\left(\eta_{i}\right)$.

Traditionally the effect of the covariates on the response $y_{i}$, is modeled by a linear predictor:

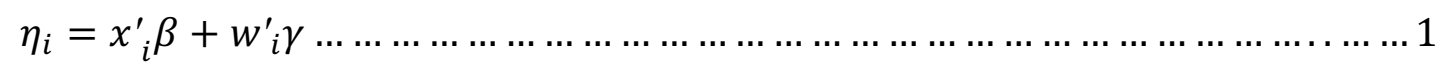

Where $\mathrm{h}$ is a known response function.

The predictor on equation 1 above is the predictor of generalized linear model. But In this Predictor, spatial variability is not included and as well as non-linearity of metrical covariate is not assumed. Therefore in order to account spatial dependency with non-linearity assumption of metrical covariate, equation (1) is extended to a geo-additive model by accommodating the spatial variability and non-linear effect of metrical covariate as follows:

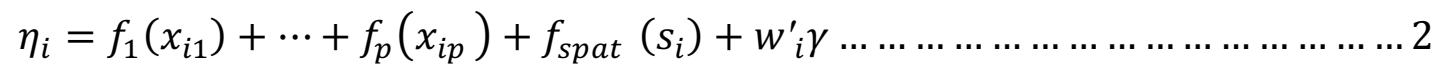

Where, $f_{1}, \ldots, f_{p}$ are nonlinear smooth effects of the metrical (continuous) covariates and $f_{\text {spat }}$ is an additional spatially correlated effect of the location $s_{i}$ an observation pertains to.

In a further step, we may split up the spatial effect $f_{\text {spat }}$ into a spatially correlated (structured) and an uncorrelated (unstructured) effect.

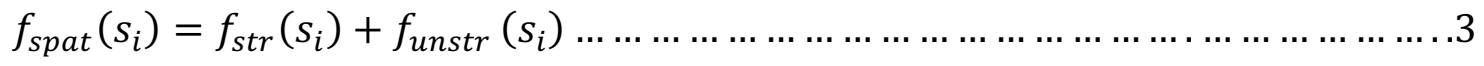

Here, spatial variability is usually contained many unobserved influences exist only locally [1]. Therefore, equation (1) is extended as the following on equation 4 and gives the predictor of geoadditive regression model.

$$
\eta_{i}{ }^{g e o}=f_{1}\left(x_{i 1}\right)+\cdots+f_{p}\left(x_{i p}\right)+f_{\text {str }}\left(s_{i}\right)+f_{\text {unstr }}\left(s_{i}\right)+w_{i}^{\prime} \gamma \ldots \ldots \ldots \ldots \ldots \ldots \ldots
$$

Where:

* $\eta_{i}$ is a linear predictor of variable of interest(prevalence of diarrhea disease ) on sociodemographic, environmental factor, behavioral risk factor and geographic variables in this study.

* $\gamma$ is unknown parameters(fixed effect parameter) corresponding categorical predictor.

* $f_{\text {str }}\left(s_{i}\right)$ is spatially correlated (structured) effect, $f_{\text {unstr }}\left(s_{i}\right)$ spatially uncorrelated (unstructured) effect. 


\section{Method of Estimation}

The type of inferential concept used for estimation of regression parameters in this study is full Bayesian inference via Markov chain Monte Carlo (MCMC).Bayesian inference is based on posterior distributions and is carried out using MCMC simulation techniques so that samples are drawn from full conditionals of single parameters or block parameters given the rest. Let $\alpha$ denote the vector of all unknown parameters in the model (i.e. $\alpha=\left(f_{j}, f_{\text {spa }}\right), \gamma$ and $\tau$ represent the vector of all variance components. Then, under usual conditional independence assumptions, for the binomial logit model:

$$
P(\alpha / y) \propto \prod_{i=1}^{n} L_{i}\left(y_{i}, \eta_{i}\right) \prod_{j}^{p}\left\{p\left(\beta_{j} / \tau_{j}^{2}\right) p\left(\tau_{j}^{2}\right)\right\} p\left(f_{\text {str }} / \tau_{j}^{2}\right) p\left(f_{\text {unstr }} / \tau_{\text {unstr }}^{2}\right) \prod_{j=1}^{r} p\left(\gamma_{j}\right) p\left(\sigma^{2}\right)
$$

Where, $\beta_{j}, \mathrm{j}=1 \ldots \ldots$, are the vectors of regression coefficients corresponding to the functions $f_{j}$.

For updating the parameters in an MCMC sampler, we used Metropolis-Hastings algorithm based on iteratively weighted least squares (IWLS) proposals introduced by [6].

\section{Results and Discussions}

\section{Moran's I and Geary's C Test Statistics for Global Spatial Autocorrelation}

The test result of global Moran's I and Geary's C indicates the presence of significant global spatial autocorrelation of Under-five diarrhea disease in Ilu Aba Bor zone (Table 1). The test results are also shown in Moran's I scatter plot (Figure 1). These global results in the distribution of Under-five diarrhea disease need to be further explored using local spatial statistics.

Table 1: Results of Global Moran's $I$ and Geary's $C$ Statistics under randomization and Normality assumption

\begin{tabular}{|l|l|l|l|l|l|l|}
\hline Assumption & Coefficient & Observed & Expected & Std Dev & $\mathbf{Z}$ & P \\
\hline Normality & Moran's $I$ & 0.33367024 & -0.07692308 & 0.16597 & 2.4739 & 0.006683 \\
\hline Normality & Geary's $C$ & 0.60338506 & 1.00000000 & 0.19983 & -1.9848 & 0.02358 \\
\hline Randomization & Moran's $I$ & 0.33367024 & -0.07692308 & 0.16797 & 2.4444 & 0.007254 \\
\hline Randomization & Geary's $C$ & 0.60338506 & 1.00000000 & 0.1947 & -2.0365 & 0.02085 \\
\hline
\end{tabular}

Significant at 0.05 levels

Hypothesis test under the global measure of spatial autocorrelation is $H_{0}$ : no spatial autocorrelation $\left(H_{0}: \rho=0\right)$ versus under the alternative hypothesis $H_{1}: \rho \neq 0$ (There is spatial autocorrelation (spatial dependence). The variance of Moran's $I$ and Geary's $C$ varies under the assumptions of normality and randomization. 
Interpretation: Based on the P-values of Moran's $I$ and Geary's $C$ coefficients, we reject the null hypothesis of no spatial autocorrelation. Furthermore, the computed Z- statistic for Moran's $I$ is positive under both Normality and Randomization assumption and Geary's $C$ is negative under both Normality and Randomization assumption indicating the existence of significant positive spatial autocorrelation (Under-five diarrhea disease clustering of similar value).

In order to visualize global spatial autocorrelation we use Moran's scatter plot under the assumptions of normality (Figure1). It shows Under-five diarrhea disease can be assumed to occur with unequal distribution at all cluster (woreda).

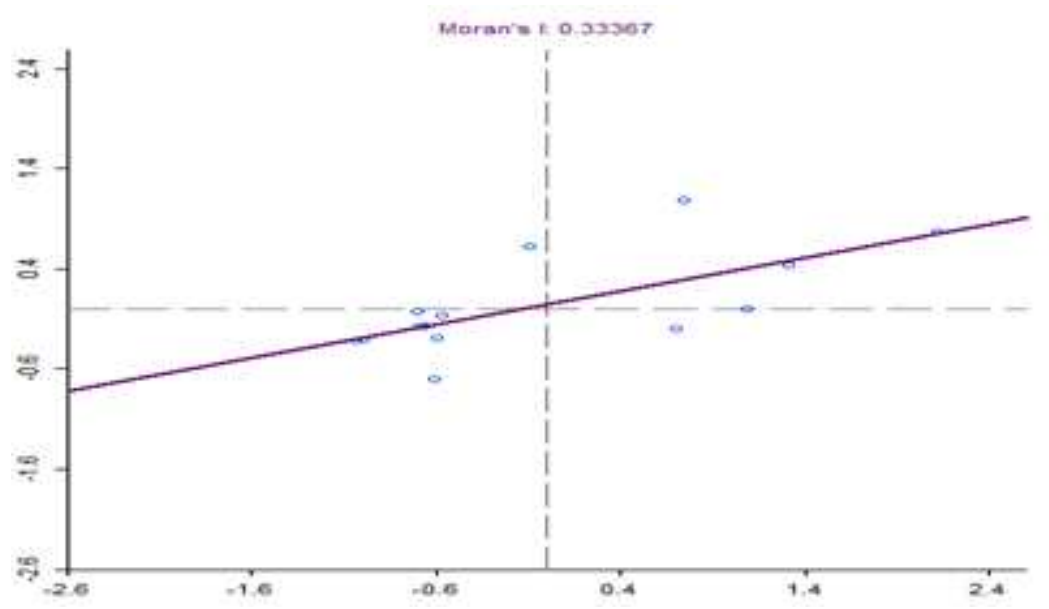

Figure 1: Moran Scatter Plot for Under-five diarrhea disease in Ilu Aba Bor Zone

From above figure 4.1 we conclude that Under-five diarrhea disease in Ilu Aba bor zone of each woreda is spatially correlated with neighboring values. From the first quadrant (upper right) of Moran scatter plot we understand that in three woredas the distribution of Under-five diarrhea disease were highly clustered. This result indicates that in these three woredas, there are high Under-five diarrhea diseases clustering of similar values (hot spots). From the 3rd quadrant (lower left) we see that the distribution of Under-five diarrhea disease in eight woredas is less clustered. This indicates that in these eight woredas the distribution of Under-five diarrhea disease are cold spots (low low). On the other hand, as it is seen from the second and fourth quadrant (lower right and upper left) of the Moran scatter plot there is Under-five diarrhea disease clustering of dissimilar values in three woreda ( either high low or low high value).

\section{Bayesian Geo-additive Regression Model result}

Table 2 below shows that the result of Bayesian geo-additive regression model which represent fixed effect of categorical covariate plus non-linear effect of metrical covariate plus spatial effect 
(both structured and un-structured spatial effect). The result showed that most of the covariates have a significant effect on the under-five child diarrheal disease. The fixed effect results of this model indicate that mother's educational level, source of drinking water, quality of toilet facility, DPT 3 vaccination, Polio 3 vaccination, and household wealth index were significantly affect the occurrence of diarrhea disease on under-five children ; since their corresponding credible interval has not included zero. In contrast of this mothers occupation, Residence place and age of child were insignificant effect on the occurrence of diarrhea disease on under-five children; since their corresponding credible interval included zero. For the convenience and get the easy understanding of the interpretation, the researchers have interpreted the exponentiation value of the coefficients of the significant variables as follows:

The coefficient value of the Source of drinkable water categories of protected was OR= exp ($0.1926)=0.82$ with $95 \%$ CI $(0.51,0.89)$. This is interpreted as children who use protected water had $82 \%$ lower odds to cause by diarrhea than those who use water from unprotected source. Concerning quality of toilet category of not good was $\mathrm{OR}=\exp (0.2456)=1.27$ with $95 \% \mathrm{CI}$ $(1.12,1.94)$, which means that children who use not good toilet facility had $27 \%$ higher odds to caused by diarrhea than those who use good toilet facility

.Regarding to DPT 3 vaccination the coefficient value for received categories was OR= exp (-$0.2556)=0.77$ with $95 \%$ CI $(0.63,0.85)$, which means that children who took DPT 3 vaccination had $77 \%$ lower odds to cause by diarrhea than those who not took.

Another finding of this study showed that educational level of mother has a significant contribution to occurrence of under-five diarrheal diseases. The odds of children who have diarrheal diseases for their mother educational level higher was 0.55 times $(\mathrm{OR}=0.55,95 \% \mathrm{CI}$ : $0.22,0.78$ ) less likely than the odds of children whose their mother has no education.

Concerning to coefficients value for Rich category of house hold wealth index $(\mathrm{OR}=0.25$, 95\%CI: $(0.11,0.62))$, which mean that, the odds of children who have diarrhea disease from rich family were 0.25 times children from poor family.

In the same way, coefficients value for Polio3 vaccination for received categories was $\mathrm{OR}=\exp$ $(-0.4058)=0.66$ with $95 \%$ CI $(0.43,0.97)$, which means that children who took Polio3 vaccination had $66 \%$ lower odds to caused by diarrhea than those who not took. 
Regarding to both metrical covariate (age of mother) and spatial effect (structural\& unstructural), they were significantly affect occurrence of under-five diarrhea disease in this study, hence their corresponding 95\% credible intervals [0.7250, 6.5865], [0.023, 3.7446], [0.01, 1.5022] respectively which does not included zero. More, the spatial and metrical covariate effect on under-five diarrhea disease were briefly explained by it is visualization using map effect and graph on section below.

Table 2: Posterior mean estimate of Bayesian Geo-additive Regression model

Variable

Category

Intercept

Mothers occupation No work(ref)

Have work

Source of drinkable Not protected(ref)

water

Protected

$-2.1640$

0.8597

$-4.0041$

$-0.6274$

quantile quantile

Quality of toilet Good (ref)

Not good

0.2456

0.2130

0.1790

0.6634

DPT3 vaccination not received (ref)

Received

$-0.2556$

0.2230

$-0.46734$

$-0.1614$

Educational level of No education(ref)

mother

Primary
Secondary

0.0167

$--0.3230 \quad 0.4670$
$-0.4215$

0.4819

$-1.2623$

0.5292 
Higher $\quad \begin{array}{llll}-0.5915 & 0.4373 & -1.4996 & -0.2530\end{array}$

$\begin{array}{llllll}\begin{array}{l}\text { House hold wealth } \\ \text { index }\end{array} & \text { Poor(ref) } & & & & \\ & & & & & \\ & \text { Medium } & 0.5547 & 0.5080 & 0.5136 & 1.4594 \\ & \text { Rich } & -1.4006 & 0.4728 & -2.2815 & -0.4798\end{array}$

Polio3 vaccination Not received (ref)

$\begin{array}{lllll}\text { received } & -0.4058 & 0.2202 & -0.8362 & -0.0298\end{array}$

Residence place $\quad$ Urban(ref)

$\begin{array}{lllll}\text { Rural } & -0.2728 & 0.2758 & -0.7988 & 0.2620\end{array}$

Smooth terms variances

\begin{tabular}{|c|c|c|c|c|c|}
\hline Age of mother & Continuous & 2.7172 & 1.5719 & 0.7250 & 6.5865 \\
\hline sx(woreda):mrf & & 0.9207 & 1.0730 & 0.023 & 3.7446 \\
\hline sx(woreda):re & & 0.2886 & 0.4090 & 0.01 & 1.5022 \\
\hline
\end{tabular}

Visualization of the effect of Metrical Covariate and Spatial effect

Figures 2, below display the posterior mean of nonlinear effect of age of mother on under-five diarrhea disease. From the pattern of this graph we have seen that the effect of mother's age is comparably higher in age interval $(<20)$, then somewhat decrease at age interval $(20-29)$ and again increase at age interval (30-39). In short, from this graph we understand that child of young mother $(<20)$ and that child of mother age $>30$ were at higher risk of diarrhea disease, as compared to mothers with age interval (20-29).

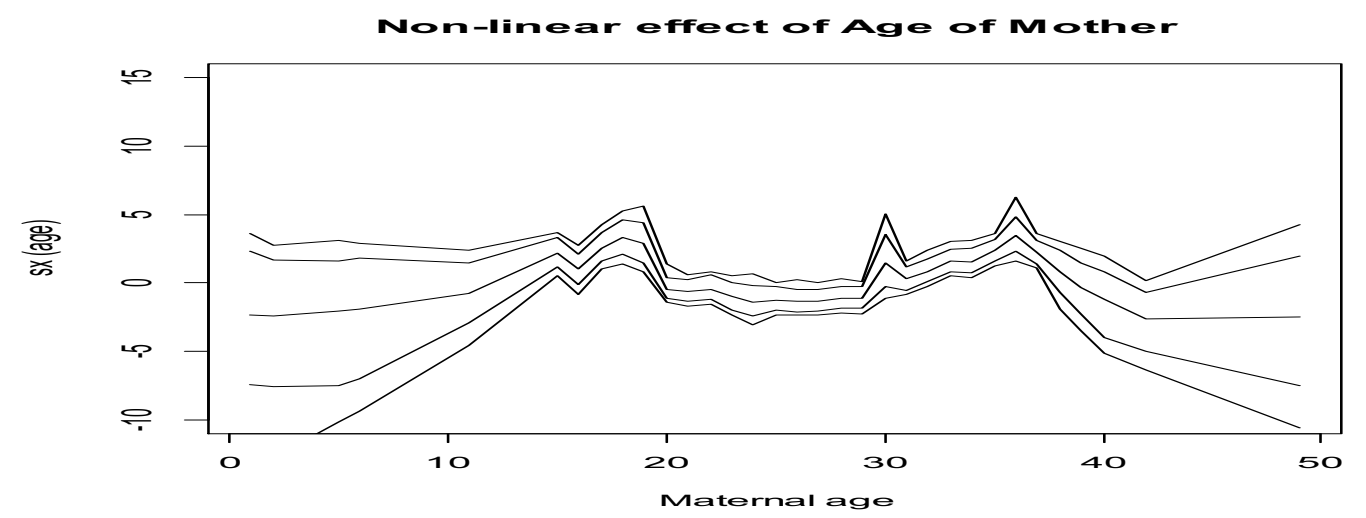

12 | $P$ a g e 
Figure 2:Non-linear effect of mother age on Under-five diarrhea disease in Ilu Aba Bor Zone With regard to spatial effects, figures 3 below depict the estimated posterior mean of the spatial effect by using map effect on the under-five diarrhea disease in Ilubabor zone. Therefore from the pattern of this map it is clearly seen that there was spatial (Geographical) variation between woredas of Ilubabor zone. The red color indicates the presence of hot spots (high-high under-five diarrhea case counts clustering) in three woredas, while the light blue color indicates the presence of cold spots (low-low under-five diarrhea case counts clustering) in the eight woredas. The pink and dark magenta color indicates clustering of dissimilar value. The map reveals that in Darimu, Metu town and Alge sachi woredas there is under-five diarrhea case counts clustering of high high values (hotspots). In Nono sale, Didu,Ale, Halu,Becho, Yayu,Doreni and Hurumu there is under-five diarrhea case counts clustering of low-low values (cold spots) is observed. On the other hand in Bilonopha there is under-five diarrhea case counts clustering dissimilar values (low-high values) and in Bure and Mettu woreda there is under-five diarrhea case counts clustering of high -low values. In general, a higher under-five diarrhea case count was observed in the northern and western part of the Ilubabor zone while the southern and eastern part has low under-five diarrhea case counts.

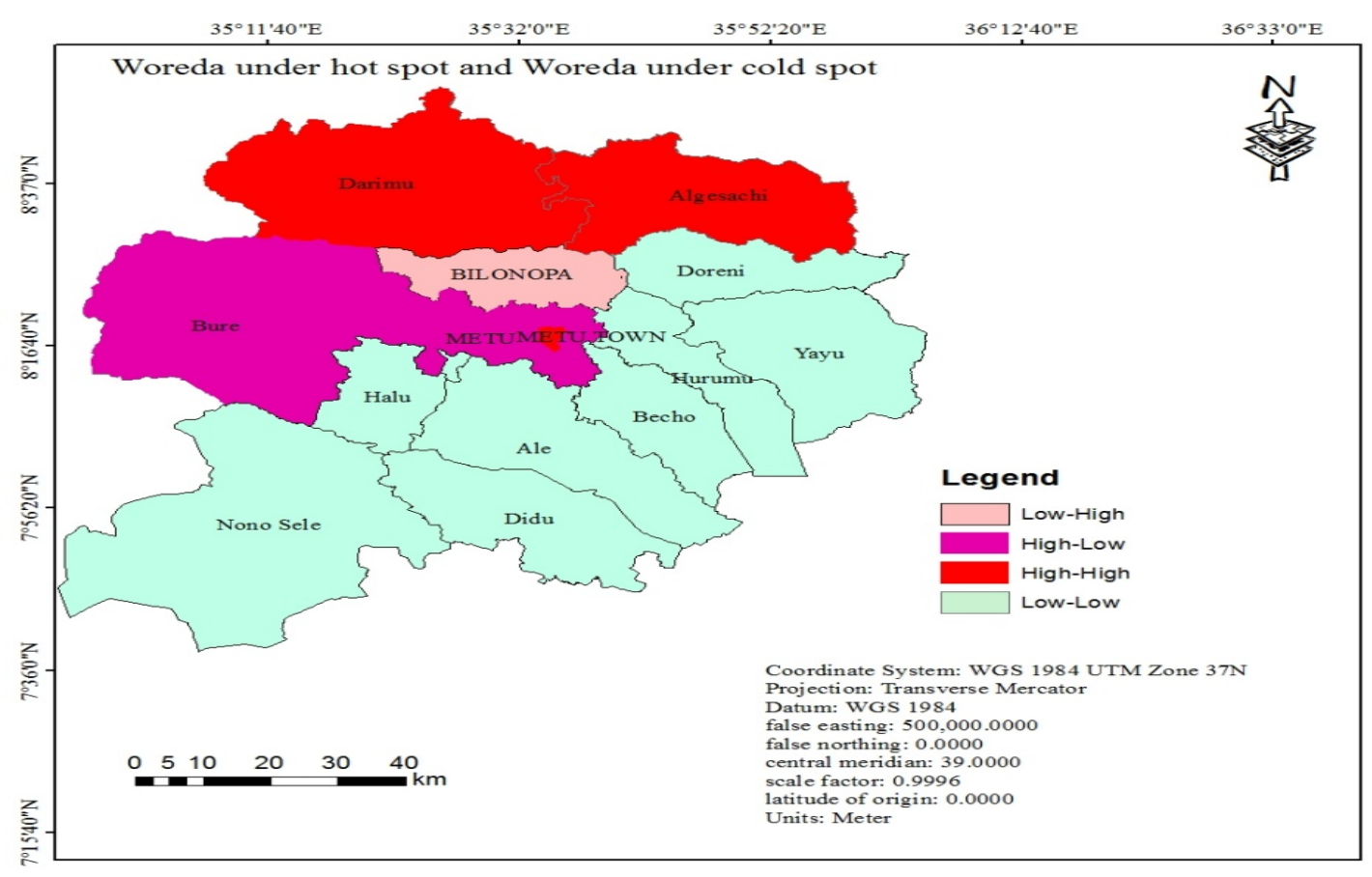

Figure 3: Spatial distribution of Under-five diarrhea disease in Ilu Aba Bor Zone 


\section{Discussions}

From explanatory spatial data analysis both global and local measure of spatial autocorrelation is used and spatial weight matrix is constructed depending on queen methods. The global tests are performed under the assumption of normality and randomization, the null hypothesis tested states spatial independence (uncorrelated of error terms) and alternative hypothesis tested states spatial dependence (correlated of error terms) for the data under consideration. Based on the Pvalues of Moran's $I$ and Geary's $C$ coefficients, we reject the null hypothesis of no spatial autocorrelation and accept alternative hypothesis of there is spatial autocorrelation. Furthermore, the computed Z- statistic for Moran's $I$ is positive under both Normality and Randomization assumption and Geary's $C$ is negative under both Normality and Randomization assumption indicating the existence of significant positive spatial autocorrelation (under-five diarrhea case counts clustering of similar values).The result of local measures of spatial autocorrelation showed that under-five diarrhea case counts in Ilu Ababor zone of each woreda is spatially correlated with neighboring woreda(cluster).Generally measures of spatial autocorrelations shows under-five diarrhea case counts in Ilu Aba bor zone of each woreda(cluster) is spatially correlated with neighboring woreda(cluster), Where high-high (hot spot) value grouping occurred and another one at the opposite extreme with low-low (cold spot) values occurred. Previous studies are also consistent with this result that under-five diarrhea disease is spatially correlated with neighboring region (cluster), where high (hot spot) value grouping occurred and another one at the opposite extreme with low (cold spot) values grouping occurred [2].

Regarding to model based data analysis part, influential factors for the under-five diarrhea disease were identified by using Bayesian Geo-additive regression model. From the fixed-effects of the model, mother's educational level, source of drinking water, place of residence, quality of toilet facility, DPT 3 vaccination, Polio 3 vaccination, and household wealth index were significantly affect the occurrence of diarrhea disease on under-five children. Concerning to Source of drinkable water, children who use protected water had $82 \%(\mathrm{OR}=0.82)$ lower odds to cause by diarrhea than those who use water from unprotected source. This result was consistent with previous study [12].

Another influential factors of under-five diarrhea disease was quality of toilet $[\mathrm{OR}==1.27]$ which means that children who use not good toilet facility had about $27 \%$ higher risk of diarrhea disease than those who use good toilet facility, which is also supported by previous study 
[7].Regarding to DPT 3 vaccination the coefficient value for received categories was ( $O R=0.77)$ which means that children who took DPT 3 vaccination had about $77 \%$ lower risk of diarrhea than those who not took. So this result was supported with previous study [8].

We also found that the odds of children who have diarrheal diseases for their mother educational level higher was 0.55 times $(\mathrm{OR}=0.55)$ less likely than the odds of children whose their mother has no education; which have the same idea with previous study [13]. Concerning to coefficients value for Rich category of house hold wealth index $(\mathrm{OR}=0.25)$, which mean that, the odds of children who have diarrhea disease from rich family were 0.25 times children from poor family. This result has the same idea with previous study [15]

In the same way, coefficients value for Polio3 vaccination for received categories was (OR $=0.66$ ) which means that children who took Polio3 vaccination had $66 \%$ lower odds to cause by diarrhea than those who not took. So this result was supported with previous study [8]

Regarding to both metrical covariate (age of mother) there was a continuous worsening of risk of under-five diarrhea disease during the age of mother less than 20 years old and between age interval (30-39) which indicate that a children with mother younger than 20 years old and age interval (30-39) were experienced high risk of diarrhea in relative to mother age interval (2029).This result has almost the same idea with previous study [10].

\section{Conclusions}

The results of this study showed that under-five diarrhea case counts in Ilu Aba Bor Zone exhibits a spatial pattern which is dependent on socioeconomic, Environmental and health factors. Under-five diarrhea case counts in the study area is significantly clustered indicating high levels in the Northern part of the zone and low levels in southern and eastern part of the zone, clustering of dissimilar value in western part of this zone.

Geographical clusters of under-five diarrhea case counts were identified through exploratory spatial data analysis, using Global Moran's $I$, Geary's $C$ and also local indicators. The results obtained reveal that the distribution of under-five diarrhea case counts in Ilu Aba Bor Zone is clustered. So our findings support the notion that under-five diarrhea disease is a basic problem of public health issue in the Ilubabor zone with spatial variation across different woredas. 
Spatial pattern of under-five diarrhea disease in Ilubabor zone was explored and the hot spot woredas were identified. This pattern suggests that the northern and western part of the zone were highly experienced with diarrhea disease.

Based on Bayesian Geo-additive regression model, mother's educational level, source of drinking water, quality of toilet facility, DPT 3 vaccination, Polio 3 vaccination, and household wealth index were significantly affect the occurrence of diarrhea disease on under-five and mothers occupation, Residence place and age of child were insignificant effect on the occurrence of diarrhea disease on under-five children.

\section{Recommendations}

The findings of this study have some important policy implications and the identification of factors those are significantly associated with under-five diarrhea disease. Depending on the above important findings, the researcher suggests the following recommendations for researchers and policymakers:

$>$ Pay special attention to health factor that affect under-five diarrhea disease distribution i.e. DPT 3 vaccination, Polio 3 vaccination.

$>$ Expand and strengthen medical facilities especially in woredas identified as hot spots.

$>$ The government and other concerned bodies should have to take attention to control the significant factors that associated with under-five diarrhea by sharing knowledge on necessity of hygiene of toilet facility and concerned body should have to do on the way of up grading the economic status and educational level of the child mother.

$>$ In addition the researchers should strongly recommend doing the same study by using spatio-temporal modeling by including time effect because of time may one the influential factors of this problem.

\section{List of Abbreviations}

AIDS: Acquired Immune Deficiency Syndrome

CI: Credible Interval

CSA: Central Statistical Agency

DIC: Deviance Information Criterion

DPT: Diphtheria, Pertussis, Tetanus

GLM: Generalized Linear Model

HIV: Human Immunodeficiency Virus 
MCMC: Markov Chain Monte Carlo

UNICEF: United Nations International Children's Emergency Fund

WHO: World Health Organization

\section{Limitations of the Study}

This study is only limited to zonal level of Ilu Aba Bor due to limited resource.

\section{Conflict of Interests}

The authors would like to declare that they have no conflict of interests in this study

\section{Acknowledgements}

The authors gratefully acknowledge Ilu Aba Bor Zone for allowing using the data. This work was financially supported by the College of Natural Science, Mettu University.

\section{Author's Contributions}

Ebsa Gelan, Muleta Worku, Azmeraw Misganaw and Dabala Jabessa conceived the idea, contributed in the design analyses completed all statistical analyses and interpretation, Ebsa Gelan, Muleta Worku, Azmeraw Misganaw and Dabala Jabessa, the corresponding author drafted the manuscript and read and approved the manuscript.

\section{Ethical Approval}

Institutional research ethics review committee (IRERC) of Mettu University obtained ethical approval. A letter of support was written to study area. Informed consent was acquired from each study participants.

\section{Funding}

None.

\section{Availability of data and materials}

The data set was taken from Ilu Aba Bor Zone Health bureau

\section{Competing interests}

The authors declare that not competing interest were available. 


\section{Reference}

1. Adebayo S, Fahrmeir L.Klasen S.(2004).Analyzing infant mortality with geoadditive categorical regression models: a case study for Niger ia. Economics and Human Biology 2: 229-244.

2. Asmamaw Atnafu, Malede MequanentSisay, Getu DebalkieDemissie and ZemenuTadesseTessema. (2019). Geographical disparities and determinants of childhood diarrheal illness in Ethiopia: FurtherAnalysis of 2016 Ethiopian Demographic and Health Survey

3. Bailey, T. C. and Gatrell, A. C. (1995). Intereactive Spatial Data Analysis, Longman, Harlow.

4. Fahrmeir, L. \&Tutz, G. (2001).Multivariate Statistical Modeling Based on Generalized Linear Models, Springer, New York.

5. Fisher Walker, L.C., Perin, J., Aryee, J.M., Boschi-Pinto, C. and Black, R.E. (2012).Diarrhea incidence in low- and middle-income countries in 1990 and 2010: A systematic review.BMC Public Health, 12.http://dx.doi.org/10.1186/1471-2458-12-220

6. Gamerman, D. (1997). Efficient Sampling from the posterior distribution in generalized linear models. Statist.Comput. 7, 57-68.

7. Godana W, Mangiste B.(2013). Environmental Factors Associated with Acute Diarrhea among Children Under Five Years of Age in Derashe District, Southern Ethiopia. Sci J Public Heal,1(3):119.

8. Hanaa Hussein.(2017).Prevalence of Diarrhea and Associated Risk Factors in Children Under Five Years of Age in Northern Nigeria: A Secondary Data Analysis of Nigeria Demographic and Health Survey 2013.

9. Kammann EE, Wand MP.(2003).Geoadditive models. Journal of the Royal Statistical Society;52:118.

10. Maria Josemere de Oliveira BorbaVasconcelosI, AneteRissinI, José Natal FigueiroaI, Pedro Israel Cabral de LiraII, Malaquias Batista Filho.(2018).Factors associated with diarrhea in children under five years old in the state of Pernambuco, according to surveys conducted in 1997 and 2006.

11. McCullagh P, NelderJA .(1989).Generalized Linear Models, $2^{\text {nd }}$ edition. Chapman \& Hall, London

12. Mengistie B, Berhane Y, Worku A.(2013). Prevalence of diarrhea and associated risk factors among children under-five years of age in Eastern Ethiopia: A crosssectionalstudy. Open J Prev Med,03(07):446-53 
13. Mohammed Eisa Abdalla1, Ahmed Elnadif Elmanssury2,3, Ahmed Eltigani Almardi2, SafaAbdallaElnour Dafaalla4.(2018).Environmental and Behavioral Factors Associated With Diarrhea Disease among Children Under Five Years Old in Mayo in Khartoum State.

14. Nelder, J. A. \&Wedderburn R. W. M. (1972).Generalized linear models. Journal of the Royal Statistical Society Series A 135, 370-384

15. Nworie, K. M., \&Aluh, D. O. (2018). Determinants of Diarrhea and Optimal Childcare among Under-Five Children in Nigeria: Insights from the 2013 Demographic and Health Survey. Fam Med MedSci Res, 7(229), 2.

16. Rosenberg M. (2007). Global Child Health: Burden of Disease, Achievements, and Future Challenges. CurrProblPediatrAdolesc Health Care. Oct;37(9):338-62.

17. UNICEF .(2016).Diarrhea remains leading killer of young children, despite the availability of simple treatment solution,updated.UNICEF.org/child-health/diarrheal disease.html

18. WHO.(2013).Diarrhoealdisease.Availablefrom:http://www.who.int/mediacentre/factsheets/fs330/en/ [Accessed7thFeb2017.UNICIF.2016.Diarrhoea.Availablefrom:https://www.unicef.org/health/index 92007.html

19. WHO.2014. Global status report on non-communicable diseases, Geneva

20. WHO. 2018. World health statistics. Monitoring health for the SDGs, sustainable development goals. Geneva 